\title{
A Case of Granulomatosis With Polyangiitis With Pulmonary Nodules and Masses
}

\author{
Qian Wu \\ Rheumatology Research Center \\ Limin Zhang \\ Shandong Provincial Hospital \\ Qingrui Yang ( $\sim$ nianqicheng2020@163.com ) \\ Shandong Provincial Hospital \\ Hongsheng Sun \\ Shandong Provincial Hospital
}

Case report

Keywords: Granulomatosis with polyangiitis, pulmonary, nodules, masses, inflammation

Posted Date: April 16th, 2021

DOI: https://doi.org/10.21203/rs.3.rs-398694/v1

License: (c) (i) This work is licensed under a Creative Commons Attribution 4.0 International License.

Read Full License 


\section{Abstract}

Background: Granulomatosis with polyangiitis (GPA) is autoimmune-mediated, necrotizing granulomatous vasculitis, primarily involving the upper and lower respiratory tract and kidneys. Pulmonary parenchymal necrosis, vasculitis, and granulomatous inflammation are the main pathological features. Pulmonary imaging visualizes various manifestations, like lung consolidation, ground-glass shadow, speckle shadow, nodules and mass shadows, cavitation, pulmonary interstitial change, pleural effusion, and multiple mediastinal lymph nodes enlargements, and stenosis or occlusion of a lobe or bronchus segment.

Case presentation: We report a 53-year-old patient diagnosed with Granulomatous polyangiitis with multiple pulmonary nodules and masses, by ultrasound-guided lung mass puncture biopsy. After treatment with methylprednisolone, Mycophenolate Mofetil, compound sulfamethoxazole and cyclophosphamide, the patient's condition improved and the pulmonary lesions were better than before.

Conclusions: In addition to specific infections and tumors, immunological diseases, especially granulomatous polyangiitis, should be considered when treating patients in a clinical setting with multiple pulmonary nodules and weights. Through close multidisciplinary cooperation and communication, the disease will be hopefully received timely diagnosis and early treatment, relieving pain for more patients.

\section{Background:}

Granulomatous polyangiitis (GPA) is a systemic inflammatory disease, which belongs to an autoimmune multisystemic small-vessel vasculitis included in the group of anti-neutrophil cytoplasmic antibody (ANCA)-associated small-vessel vasculitides (1). It presents a broad spectrum of manifestations, from common respiratory and neurological symptoms, to infrequent cardiac complications, and remains one of the most challenging diagnostic dilemmas in clinical medicine today (2). The main manifestations of lower airways include cough, breathlessness, stridor, wheeze, small airway obstruction, pulmonary nodules, cavitating lung lesions, pleuritis, pleural effusions, pulmonary infiltrates, pulmonary hemorrhage, alveolar capillaritis, and respiratory failure (1).

\section{Case Presentation:}

A 53-year-old male was admitted to the hospital on June 25, 2018, due to "Cough and sputum repeatedly for more than four years and headache for two months."

At first, he admitted to a municipal hospital, and cerebral MRI identified slight focal brain ischemic and rhinitis. Double lung CT identified multiple nodules and soft tissue shadows, and bilateral pleural thickening (Fig. 1A, 1B, 1C, 1D). The doctor gave the patient the treatment of anti-infection, relieving cough and reducing sputum, after which symptoms improved from admission. However, in order to clarify the diagnosis, he was admitted to our hospital again. 
We considered that the preliminary diagnosis was pulmonary inflammation or cancer. Thus, we made differential diagnoses based on the following aspects: (1) Pulmonary infection: The patient had no history of long-term hormone or broad-spectrum antibiotic use, and did not belong to the population with low immunity, had no fever, no night sweats, no cough or yellow sputum, no cough with odorous sputum, and laboratory examination showed procalcitonin, EB virus-DNA, CMV virus-DNA, fungal 1,3 D-glucan detection, fungal galactomannan detection, sputum bacteria and fungi cultivation, sputum smear with acid-fast bacilli were all negative. Therefore, we found no clear evidence of pulmonary pathogenic infection. (2) Connective tissue disease: The patient presented with cough, white sputum, ESR: $72 \mathrm{~mm} / \mathrm{H}$, CRP: $85.67 \mathrm{mg} / \mathrm{L}$, Antiprotease 3: $200 \mathrm{RU} / \mathrm{mL}$, C-ANCA 1:10 positive, IgG4 $4500 \mathrm{mg} / \mathrm{L}$, thus, we thought that systemic vasculitis was to be more likely to recur. (3) Lung tumors: The lung tumor markers of the patient, including carcinoembryonic antigen, carbohydrate antigen 50, carbohydrate antigen 125 , carbohydrate antigen 242 , squamous cell carcinoma antigen were all negative.

However, to further clarify the diagnosis and communicate with the patients, a B-ultrasound-guided right lung biopsy was performed according to the suggestion of imaging specialists. The pathology displayed that right lung biopsy with chronic inflammation and fibrogenic hyperplasia. Immunohistochemical results: CD68+ (diffuse), SMA-, STAT6-, desmin-, S100-, CD34-, Bcl-2, ALK-, Ki67-, IgG-, IgG4- (Fig. 2). So, we did not think about lung cancer. According to the 2017 ACR/EULAR (American College of Rheumatology/ European Alliance against Rheumatism) granulomatous vasculitis classification criteria, patients with positive C-ANCA $=5$ points, and patients with pulmonary imaging indications of the nodule, mass, or cavity formation $=2$ points.

Therefore, the patient with a total of 7 points, and a total score of 5 or more points was diagnosed with granulomatous vasculitis and treated with $80 \mathrm{mg}$ methylprednisolone iv drip QD, Mycophenolate Mofetil $0.5 \mathrm{~g}$ PO bid, compound sulfamethoxazole one-piece PO TID, and cyclophosphamide $0.4 \mathrm{~g}$ iv drip stat. After treatment for ten days, we reviewed the following indicators: White blood cell $9.25 \times 10 * 9 / \mathrm{L}$ increased to $16.33 \times 10 * 9 / \mathrm{L}$, hemoglobin $103 \mathrm{~g} / \mathrm{L}$ increased to $125 \mathrm{~g} / \mathrm{L}$, platelet $510 \times 10 * 9 / \mathrm{L}$ decreased to $400 \times 10 * 9 / \mathrm{L}$, ESR $72 \mathrm{~mm} / \mathrm{H}$ decreased to $27 \mathrm{~mm} / \mathrm{H}$, CRP $85.67 \mathrm{mg} / \mathrm{L}$ decreased to $0.87 \mathrm{mg} / \mathrm{L}$. A reexamination of lung CT showed multiple patchy and blocky high-density shadows in both lungs, with multiple cords around them, local pleural thickening and pulling, cavities in some of the lesions, bands of slightly high-density shadows in the hollow of the left lower lobe, local bronchiectasis, and thickening of the bronchial wall. Compared with a CT from another hospital on June 15, 2018, the lesions were smaller to different degrees (Fig. 3).

\section{Discussion And Conclusions:}

Almost all GPA patients present with either upper airway or lower respiratory tract lesions (3). Types of GPA pulmonary lesions fall within a broad spectrum (3). The most commonly detected lesion types were nodules and masses, which most widely measured between $20-40 \mathrm{~mm}$, varied in size, and buds may be located in all areas and are usually multiple and bilateral (4). Consolidation and thickening of the 
bronchial wall were also observed, including cavitation, necrosis, speculation, and invasion of the fissure, pleura, or diaphragm (4-6).

The process of pathetic necrosis is fundamental to the development of extravascular and vascular lesions (7). The microscopic hallmark of GPA is a triad of parenchymal disease, vasculitis, and granulomatous inflammation $(5,8,9)$. Low power views of necrosis identified large areas with geographic decay that appeared basophilic, termed "blue necrosis." High power views of these necrotic zones showed microabscesses with neutrophilic and nuclear debris. The vasculitis of GPA impacts small vessels under $5 \mathrm{~mm}$ in diameter, including arterioles, venules, and capillaries. The vessel walls frequently exhibit chronic inflammatory infiltrates, neutrophil, fibrinoid, transmural necrosis, and granulomas (5).

GPA treatment includes remission-induction therapy and maintenance therapy. For remission-induction therapy, all cases require an initial prednisone dose of $1 \mathrm{mg} / \mathrm{kg} / \mathrm{d}$ for 2 to 4 weeks, with a remissioninduction time of approximately 3-6 months. Severe cases (The Five-Factor Score, FFS $\geq 1$ point), especially those with renal and pulmonary involvement requiring hyper oxygen or mechanical ventilation, can be given methylprednisolone $7.5-15 \mathrm{mg} / \mathrm{kg} / \mathrm{d}$ for $1-3$ days at initial treatment $(10,11)$.

Cyclophosphamide, combined with GC, has been used since the 1970 s to control severe vasculitis and maybe the first choice for induction. The cyclophosphamide dose is $0.5-0.7 \mathrm{~g} / \mathrm{m} 2$ body surface area, or $15 \mathrm{mg} / \mathrm{kg}$ intravenous impingement, and administered at day $0,14,28$, and once every three weeks after that until the disease was in remission. Patients aged $>65$ years will receive a fixed dose of $500 \mathrm{mg}$ per dose. Combined with sodium, methamphetamine can reduce bladder toxicity. The oral dose is 1-3 $\mathrm{mg} / \mathrm{kg} / \mathrm{d}$, or $200 \mathrm{mg}$ QOD for 3-6 months, and $1 \mathrm{mg} / \mathrm{kg} /$ day after stabilization for 1 or several years (1012).

CD20 monoclonal antibodies have been used since 2011 to induce remission and treat severe, recurrent vasculitis. The treatment dose is $375 \mathrm{mg} / \mathrm{m} 2 /$ week for four weeks, or $1 \mathrm{~g}$ every two weeks, for four weeks. RAVE (Rituximab in anti-neutrophil Cytoplasmic Antibody Associated with Vasculitis) tests show that Rituximab is not superior to cyclophosphamide when considering induction of remission. The main adverse reactions included anaphylaxis, infection, decreased immunoglobulin $\mathrm{G}(\mathrm{IgG})$ and immunoglobulin M during infusion (10-12).

In severe alveolar hemorrhage requiring mechanical ventilation, plasmapheresis contributes to the restoration of organ state and function. As adjuvant therapy for GC or CTX, enhanced plasmapheresis is feasible at the initial stage of induction, that is, one time per day for seven days, $2-3 \mathrm{~L}$ of plasma per exchange, after which the interval time extended (10-12).

Maintenance therapy lasts for 12 to 24 months. After pulse therapy of glucocorticoids, the dose should be reduced to $0.5-1 \mathrm{mg} / \mathrm{kg} / \mathrm{d}$ for at least four weeks, gradually reduced to $12.5-20 \mathrm{mg} / \mathrm{d}$ for three months after that, and reduced to $5 \mathrm{mg} / \mathrm{d}$ for six months. As for other drugs, including Mycophenolate Model, Azathioprine, Methotrexate, Leflunomide, or Rituximab for maintenance therapy (10-12). 
Furthermore, GPA patients should take compound sulfamethoxazole (2-6 tablets/d) regularly to prevent pneumocystis carinii pneumonia. Long-term use can reduce GPA recurrence and prolong survival.

The leading causes of recurrence were improper dosage or course of GC and cytotoxic drugs, co-infection, and significant stress.

Thus, we report a rare case of Granulomatous polyangiitis with multiple pulmonary nodules and masses like lung cancer as well as infection, which showed a good response after immunosuppressive therapy. Therefore, respiratory specialist and oncologists should be aware of the possibility of GPA when diagnosing patients with multiple pulmonary lesions.

\section{Declarations:}

\section{Ethical Approval and Consent to participate}

This study was approved by the Review Board for Shandong Provincial Hospital Affiliated to Shandong First Medical University, in Jinan, P.R.China.

\section{Consent for publication}

Written informed consent was obtained from the patient for publication of this case report and accompanying images.

\section{Availability of supporting data}

The dataset supporting the conclusions of this article is included within the article.

\section{Competing interests}

The authors declare that they have no competing interests.

\section{Funding}

This work was supported by the grant No. 81501399/H1008 from National Natural Science Foundation of China (CN).

\section{Acknowledgements}

Not Applicable.

\section{Authors' contributions}

QW and Lm Z prepared the manuscript and the literature search; QW reviewed and edited the manuscript; Hs S corrected and revised the manuscript; QW and Qr Y treated and observed the patient; Hs S and Qr Y reviewed the manuscript. All authors read and approved the final manuscript. 


\section{References:}

1. Greco A, Marinelli C, Fusconi M, Macri GF, Gallo A, De Virgilio A, et al. Clinic manifestations in granulomatosis with polyangiitis. Int J Immunopathol Pharmacol 2016;29:151-9.

2. Grygiel-Górniak B, Limphaibool N, Perkowska K, Puszczewicz M. Clinical manifestations of granulomatosis with polyangiitis: Key considerations and major features. Postgrad Med 2018;130:581-96.

3. Homma S, Suzuki A, Sato K. Pulmonary involvement in anca-associated vasculitis from the view of the pulmonologist. Clin Exp Nephrol 2013;17:667-71.

4. Guneyli S, Ceylan N, Bayraktaroglu S, Gucenmez S, Aksu K, Kocacelebi K, et al. Imaging findings of pulmonary granulomatosis with polyangiitis (wegener's granulomatosis): Lesions invading the pulmonary fissure, pleura or diaphragm mimicking malignancy. Wien Klin Wochenschr 2016;128:809-15.

5. Scapa JV, Fishbein GA, Wallace WD, Fishbein MC. Diffuse alveolar hemorrhage and pulmonary vasculitides: Histopathologic findings. Semin Respir Crit Care Med 2018;39:425-33.

6. Cordier JF, Valeyre D, Guillevin L, Loire R, Brechot JM. Pulmonary wegener's granulomatosis. A clinical and imaging study of 77 cases. Chest 1990;97:906-12.

7. Mark EJ, Matsubara O, Tan-Liu NS, Fienberg R. The pulmonary biopsy in the early diagnosis of wegener's (pathergic) granulomatosis: A study based on 35 open lung biopsies. Human Pathology 1988;19:1065-71.

8. Travis WD, Hoffman GS, Leavitt RY, Pass HI, Fauci AS. Surgical pathology of the lung in wegener's granulomatosis. Review of 87 open lung biopsies from 67 patients. Am J Surg Pathol 1991;15:31533.

9. Lombard CM, Duncan SR, Rizk NW, Colby TV. The diagnosis of wegener's granulomatosis from transbronchial biopsy specimens. Hum Pathol 1990;21:838-42.

10. Terrier B, Guillevin L. Treatment of pulmonary vasculitis. Semin Respir Crit Care Med 2018;39:504-10.

11. Geetha D, Jefferson JA. Anca-associated vasculitis: Core curriculum 2020. Am J Kidney Dis 2020;75:124-37.

12. Lutalo PM, D'Cruz DP. Diagnosis and classification of granulomatosis with polyangiitis (aka wegener's granulomatosis). J Autoimmun 2014;48-49:94-8.

\section{Figures}



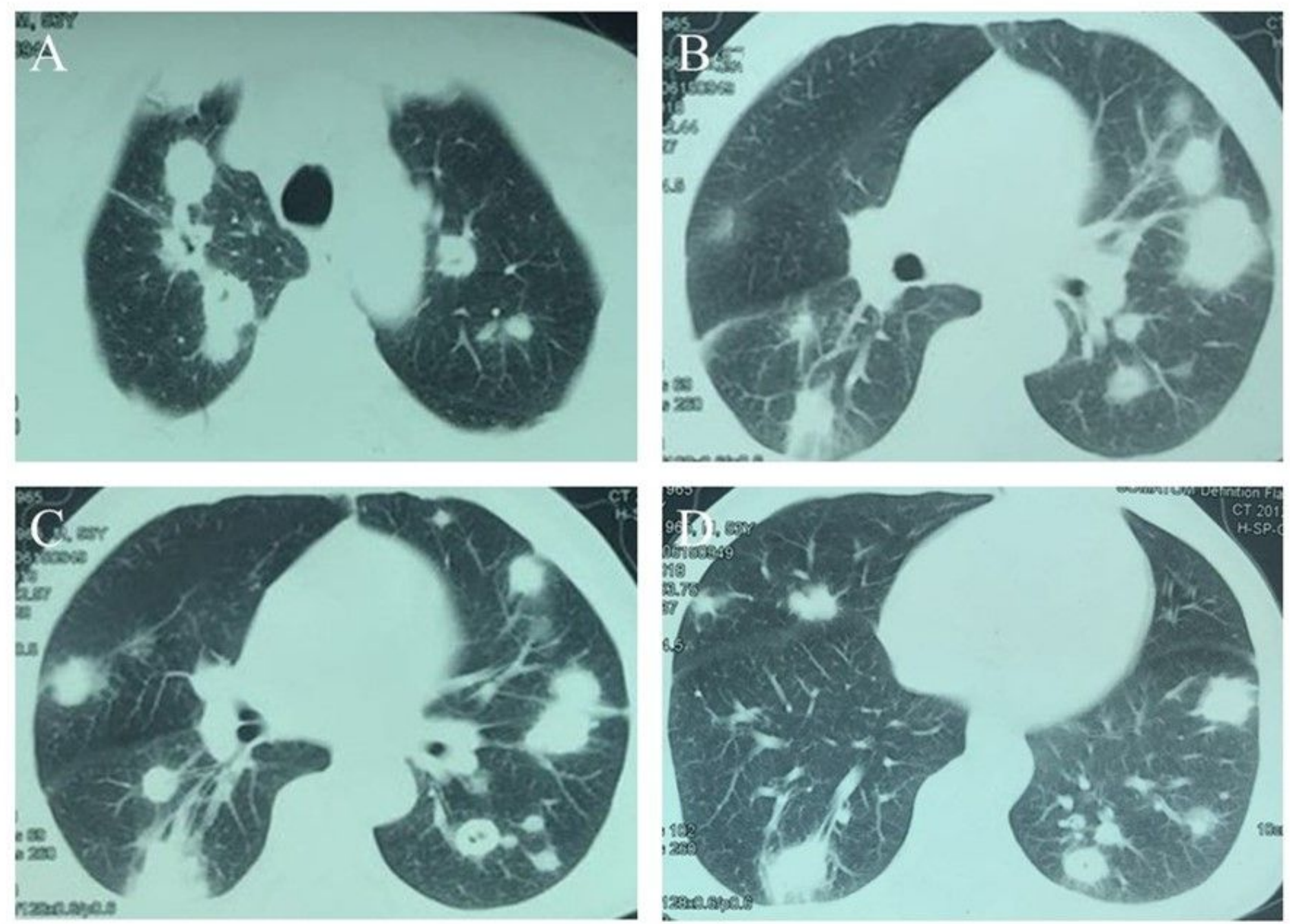

\section{Figure 1}

Multiple nodules and soft tissue shadows in both lungs, fungal infection and granuloma could not be ruled out, local thickening of bilateral pleura 

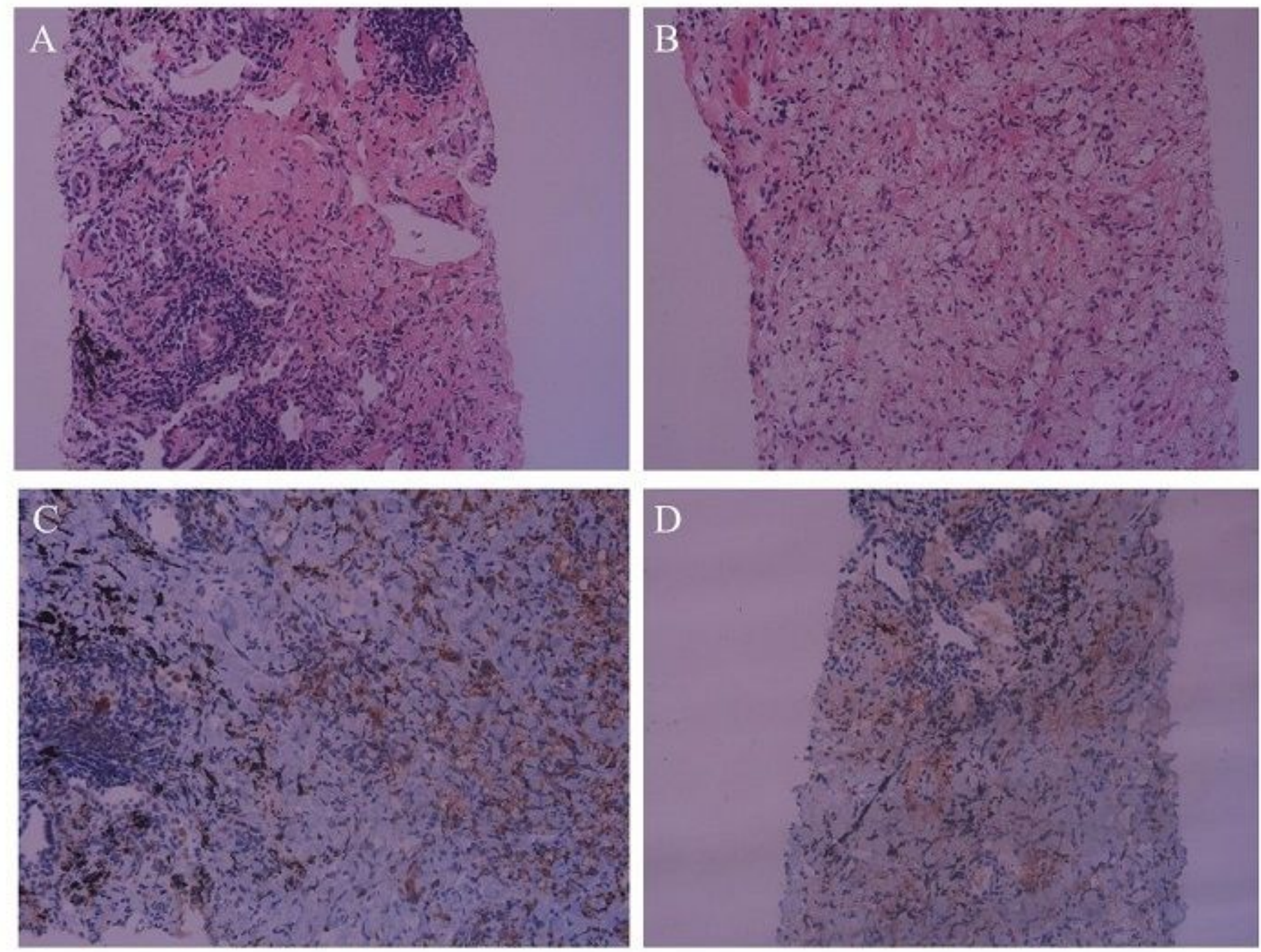

Figure 2

A. inflammatory cell infiltration -HE (100 fold) B. Histiocytosis -HE (100 fold) C. CD 68+ tissue cells (400 fold) D. IgG4 negative (100 fold) 

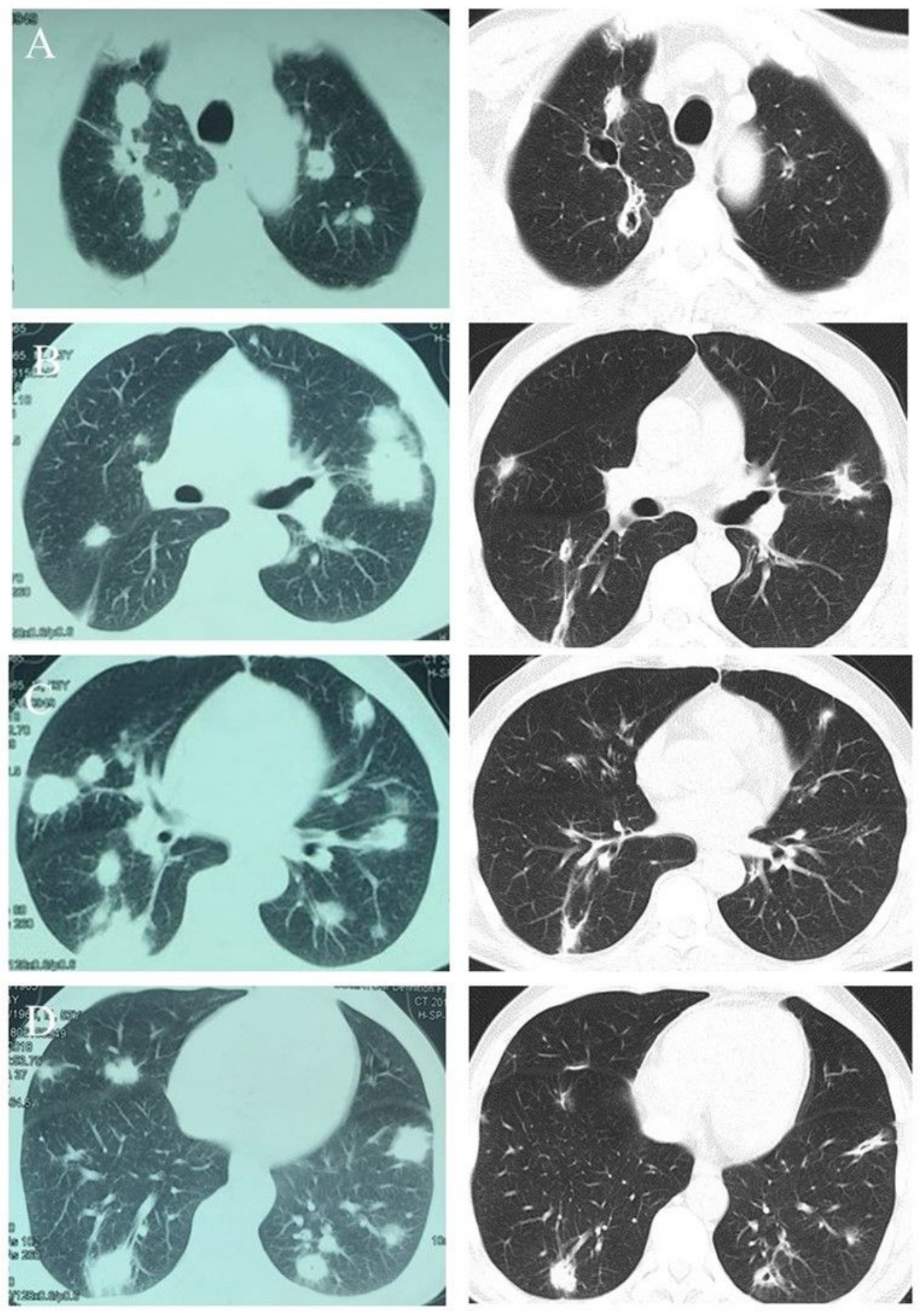

Figure 3

Compared with pulmonary CT of June 15,2018 , the lesions were smaller in different degrees. 